REVISTA E. UT:AIÓN
Revista Educación

ISSN: 0379-7082

ISSN: 2215-2644

revedu@gmail.com

Universidad de Costa Rica

Costa Rica

\title{
Condiciones de la infraestructura educativa en la región pacífico central: los espacios escolares que promueven el aprendizaje en las aulas
}

\author{
Quesada-Chaves, María José \\ Condiciones de la infraestructura educativa en la región pacífico central: los espacios escolares que \\ promueven el aprendizaje en las aulas \\ Revista Educación, vol. 43, núm. 1, 2019 \\ Universidad de Costa Rica, Costa Rica \\ Disponible en: http://www.redalyc.org/articulo.oa?id=44057415023 \\ DOI: https://doi.org/10.15517/revedu.v43i1.28179
}

Esta obra está bajo una Licencia Creative Commons Atribución-NoComercial-SinDerivar 3.0 Internacional. 


\section{Condiciones de la infraestructura educativa en la región pacífico central: los espacios escolares que promueven el aprendizaje en las aulas}

School infrastructure in the central pacific region of costa rica: designs that promote learning in the classroom

María José Quesada-Chaves

Universidad de Costa Rica, Costa Rica

majosequesada@gmail.com

iD http://orcid.org/0000-0002-2901-2297
DOI: https://doi.org/10.15517/revedu.v43i1.28179

Redalyc: http://www.redalyc.org/articulo.oa?id=44057415023

Recepción: 23 Enero 2018

Aprobación: 01 Diciembre 2018

\section{Resumen:}

Esta investigación tiene como objetivo evaluar el estado actual de las instalaciones físicas de las aulas en las escuelas y colegios públicos de la Región Pacífico Central y analizar su incidencia en el ámbito socioemocional para favorecer el verdadero aprendizaje. El enfoque de investigación utilizado fue mixto de tipo exploratorio y descriptivo tanto de los componentes físicos de la arquitectura del entorno escolar como de los aspectos relacionados con la estética del aula que repercuten directamente en el aprendizaje. Para efecto del presente estudio, se analizaron 33 escuelas y colegios públicos de la zona bajo investigación. Los instrumentos utilizados en la recopilación de la información fueron la observación no participativa y cuestionarios a administradores educativos, profesores y alumnos. La información obtenida se considera un acertado insumo para las autoridades educativas de la región y educadores a fin de reflexionar y promover un mejoramiento continuo de los espacios educativos utilizados en cada centro de estudios.

Palabras Clave: Infraestructura educativa, Aula escolar, Espacios educativos, Estética del aula.

\section{ABSTRACT:}

The objective of this article is to assess the current state of classrooms pertaining to the public primary schools and classrooms in the Central Pacific Region of Costa Rica and, furthermore, analyze their socio-emotional impact in promoting a meaningful learning environment. A total of 33 schools were analyzed as part of this study. The data collection instruments that we used were non-participatory, observation and questionnaires answered by school administrators, teachers and students. Research approaches were exploratory and descriptive with regards to the describing school architecture and classroom design aesthetics that directly influence emotional behavior and learning. The information collected serves as useful input for both school authorities and teachers to be able to reflect raise awareness and enhance their classroom designs to promote classrooms that are meaningful learning spaces.

KEYWORDS: Educational infrastructure, School buildings, Classrooms, Classroom designs, Classroom aesthetics.

\section{INTRODUCCIÓN}

La necesidad de mejorar la calidad estética y las condiciones de infraestructura de los ambientes educativos debería ser una prioridad de las políticas educativas con el fin de crear una atmósfera óptima que promueva los procesos de enseñanza y aprendizaje para promover el sentido mismo de la educación más allá de sus propósitos académicos. Con el presente estudio se busca analizar la relación que existe entre espacios escolares y las experiencias significativas que se deben llevar a cabo dentro de las instituciones educativas. La educación se ha dejado en segundo plano, a la hora de no mejorar las políticas definidas por parte del gobierno para apoyar a las instituciones educativas para mejorar condiciones básicas de estética educativa (luz, ruido, ventilación), más aún se tiene un sistema que atrasa y complica la inversión de recursos destinados para

Notas DE AUTOR 
mejorar las condiciones de los centros educativos. Es evidente apreciar que la calidad de la infraestructura y estética de las escuelas y colegios se han visto relegadas frente a instituciones públicas como los bancos, por ejemplo, donde la estética dista en gran medida de la de los centros educativos. Las entidades bancarias se caracterizan por poseer una infraestructura cuidada estéticamente, elaborada con materiales lujosos y muy agradables a la vista. Las casas de enseñanza del sector público en cambio son lugares poco acogedores construidos con materiales pobres que desmotivan al estudiantado. Esta situación queda en evidencia cuando se analiza por ejemplo la cantidad de presupuesto que se invierte en remodelaciones bancarias ante la dificultad que posee el Ministerio de Educación Pública de Costa Rica para invertir en infraestructura educativa.

$\mathrm{Al}$ respecto, en la noticia Escuelas y colegios con pésima infraestructura pese a disponer de $\$ 150$ mil millones (Cordero, 2018), se ejemplifica la situación que han vivido tres instituciones educativas que se encuentran en condiciones deplorables e insalubres. Resaltan la ineficiencia que caracteriza al MEP para poder invertir en infraestructura, específicamente a la División de Infraestructura Educativa, la coordinadora académica del Napoleón Quesada, Noemy Urtecho, afirma que "la DIEE del Ministerio de Educación Pública es una de las principales culpables de esta situación, pues sus funcionarios han puesto muchas trabas en el camino" (Cordero, 2018, párr. 16). Continúa la nota con la percepción del Director de uno de los Centros Educativos en mención indicando que en Costa Rica los procesos de construcción son complicados debido a la gran cantidad de trámites y permisos que se ven involucrados en el proceso.

Por otro lado, y de acuerdo con una noticia Bancos invierten más para tener operaciones sostenibles (Fallas, 2016), el sistema bancario nacional ha decidido apostar por inversión en obras de infraestructura amigable con el medio ambiente, esto con el fin de mejorar las condiciones actuales de los inmuebles.

Desde 2008, el BCR toma medidas para disminuir el impacto de sus operaciones en el ambiente. En este proceso destaca la incorporación de parámetros de construcción sostenible cuando se desarrollan remodelaciones y construcciones nuevas. Incluyen el uso de luces LED, vidrio temperado y laminado con control térmico, implementos con porcentaje de material reciclado y sistemas de aire acondicionado que consume únicamente la energía necesaria. También en los edificios remodelados se reutilizó hasta un 60\% de la estructura original para reducir el impacto del proceso constructivo y, por ende, la huella de carbono del proceso. (Fallas, 2016, párr. 6).

De esta manera, se logra comprobar cómo distan ambas realidades, por un lado se tienen centros educativos que no reúnen las condiciones mínimas de infraestructura, en muchos casos se pone en riesgo tanto a los estudiantes como a los docentes, pues no se cumple con la normativa en cuanto a accesibilidad. Y, por otro lado, se tiene un sistema bancario que puede invertir en instalaciones más modernas, tecnológicas y amigables con el ambiente con el fin de reducir en gran medida la huella de carbono.

Los elementos que componen el ambiente escolar son muchos y ninguno se puede dejar de lado, pues todos en su conjunto son igual de importantes. La iluminación, los colores, el material didáctico que se despliega en los muros de las aulas, las zonas verdes, la higiene y el aseo son algunos de los factores que se ven inmersos en la estética del aula. Todos estos juegan un rol sumamente importante, tal y como lo plantea Delgado (2009):

A medida que se avanza en el sistema educativo, la estética de las aulas (ornamentación, ambiente acogedor...) cada vez se descuida más. En Educación Infantil las aulas están bien iluminadas y adornadas, sin embargo, al ascender a las aulas de Bachillerato, estas denotan deterioro causado por el uso y descuido (p.141).

Cabe señalar que la niñez y la juventud invierten gran cantidad de horas dentro de las instituciones educativas, el rendimiento académico y la disciplina del aula se verían favorecidos si el ambiente físico fuera más atractivo e interesante para la población estudiantil. Suárez Palos, citado por Delgado (2009) afirma:

Tanto los profesores como los alumnos pasan gran cantidad de tiempo en el aula, por lo tanto, es conveniente crear un ambiente en el que se sientan a gusto, cómodos y seguros, facilitando con todo ello el proceso de desarrollo, socialización y creación (p.152). 
En la Región Pacífico Central existen instituciones que se encuentran en condiciones precarias, que lejos de promover la creatividad y el desarrollo de competencias o de formar individuos más críticos, se está induciendo indirectamente a que los estudiantes vean la institución educativa como un lugar aburrido y, por ende, se desmotivan al asistir a clases, incluso lo toman como un castigo porque es más interesante estar en otros sitios antes de ir a la escuela o al colegio, lo cual en muchos casos ayuda a incrementar el índice de deserción escolar.

\section{REFERENTE TEÓRICO}

\section{Espacio escolar}

El concepto de espacio escolar debe visualizarse como un espacio estéticamente agradable donde se motive al estudiante a vivenciar un proceso de enseñanza y aprendizaje exitoso. De acuerdo con Viñao (1993) "se entiende por edificio escolar el ambiente físico educativo propicio para formar integralmente al alumno" (p.30). Por esta razón, si lo que va a albergar el edificio escolar es a niños y jóvenes, estos deben estar atractivamente diseñados para satisfacer las necesidades y expectativas del estudiantado tales como: regulación del ruido dentro de la institución educativa, iluminación y ventilación apropiada dentro del aula, especialmente en la zona Pacífico Central donde el clima es cálido. Además, los estudiantes necesitan tener espacios dentro de la institución educativa que les permita desplazarse, jugar, interactuar con los compañeros y para eso se necesita de una infraestructura facilitadora de dicha función. Es sabido que los menores cuentan con amplia variedad de opciones para el ocio, en su mayoría espacios coloridos, dinámicos, amplios, por eso la escuela no debe apartarse de lo que el estudiante espera encontrar dentro del aula. Continúa Viñao (1993):

Con el diseño del edificio escolar es obvio que toda escuela es para el niño. El maestro, el profesional que diseña el aula de clase, la ubicación, forma, textura, el color del edificio; todo tiene que acomodarse a la realidad dinámica del educando y no a las normas convencionales del adulto, educador o arquitecto (p.31).

Debe entonces contemplarse a la hora de diseñar espacios escolares, la realidad y la percepción que tengan los estudiantes y no basarse en un momento específico, pues al ser un espacio de larga duración, debe entenderse, como lo menciona el autor, el aula como un espacio el cual debe construirse para albergar seres cambiantes, dinámicos y sobre todo individuos en proceso de formación. Por sí solo, el ambiente de aula y el diseño del edificio escolar realiza un papel determinante, muchas veces de éxito o fracaso de un proceso educativo:

Ejerce un influjo educativo o deseducativo, real, aunque intangible en el psiquismo de los alumnos que lo usan y, por consiguiente, la arquitectura escolar es un medio para educar, y el arquitecto empieza a educar desde la mesa de dibujo (Viñao, 1993, p.31).

De acuerdo con Sanz (2016) las condiciones estéticas deben ser agradables, amigables y sobre todo acogedoras. Es necesario que exista una armonía entre el elemento y el uso que se le dé, pues los recursos y factores estéticos juegan un rol importante y este ha de ser acompañado por una buena guía. Al respecto, el autor establece:

No solo los condicionantes estéticos determinan las preferencias del alumnado. Los usos y costumbres que se hacen de los entornos, vinculados a determinadas actividades pedagógicas, también condicionan la predilección o la antipatía. De manera que los contenidos mostrados en un tablón, el tipo de juegos de un rincón, o la presión que supone salir a la pizarra, puede hacer aborrecer determinados rincones (p.58).

Es importante entonces que el docente sepa hacer buen uso del espacio, especialmente en aulas infantiles donde la escuela debe verse como el primer lugar ajeno al hogar donde los niños se relacionan y construyen conocimientos, Fernández (1997) plantea: 
El espacio de la escuela bien entendido viene a ser como el lugar donde se entrenan las primeras miradas, gran desafío para lo reducido que resulta a veces la respuesta formal de la arquitectura en tantos centros y recintos escolares donde acontece la formación y el desarrollo de las actividades pedagógicas en los primeros años del aprendizaje (p.516).

El problema ha radicado en que se ha minimizado el poder del edificio escolar y la influencia que este ejerce en los procesos educativos y es que el espacio tiene una función didáctica. Cabe señalar que "cualquier deficiencia en el número o tamaño de los espacios disponibles para la enseñanza puede afectar negativamente la conveniencia educativa de una escuela" (Castaldi, 1974, p.87). Al respecto, Santos (1993) plantea que "vivimos y trabajamos en las escuelas sin percatarnos de la influencia que los espacios tienen en los miembros de la comunidad escolar" (p.1).

Se plantea un discurso donde se le da gran importancia al aprendizaje significativo, pues se habla que a través de la educación se deben formar ciudadanos críticos, quienes promuevan el cambio y mejoren la calidad de vida de la sociedad actual, sin embargo, "la rigidez en la utilización del espacio ha inducido a comportamientos docentes/discentes monolíticos y, a veces, contradictorios con los principios didácticos” (Santos, 1993, p.1). Lo cual es una situación lamentable porque se tienen espacios escolares dedicados muchas veces a la transmisión per se de conocimientos o contenidos, alejándose de formar individuos críticos capaces de poder reflexionar y actuar de manera efectiva en el mundo globalizado en el que se desarrollan.

Todos los espacios físicos ejercen una influencia directa en los individuos que interaccionan en él, en especial si hablamos de los niños. Para Viñao (1993) "la influencia del medio en el psiquismo del niño deja profundas huellas, ya que es el primer lugar donde entra en contacto permanente con otras personas ajena a su familia" (p. 32). Es en la escuela donde este experimentará y vivirá situaciones que involucren sentimientos tanto positivos como negativos; es decir, vivirá una adaptación o desadaptación dentro de su desarrollo como ser humano. Santos (1993) al respecto afirma que "en el centro escolar se viven muchas horas, se comparten muchas actividades y se establecen muchas relaciones” (p. 2). De igual manera Viñao (1993) indica:

Es necesario que el ambiente de la escuela, sea agradable para darle al niño un clima de seguridad, el cual puede garantizarse al máximo por el simple hecho de estar visualmente, en contacto con el mundo exterior, con la precisión de las condiciones acústicas, con la convivencia de los volúmenes y colores del conjunto en una construcción, ubicada en un marco de armonía (p.33).

Debe entonces valorarse desde esta perspectiva la importancia e influencia que ejerce el edificio escolar en procesos educativos con el fin de poder crear ambientes seguros, cómodos, los cuales motiven y permitan al estudiante sentirse a gusto para adquirir nuevos conocimientos. Este ambiente se logra solamente si existe un equilibrio entre los factores que se relacionan con la estética, como lo son el factor pedagógico y el arquitectónico, tal y como lo expone Sanz (2016).

En relación con los factores estéticos determinantes de la calidad y el confort en la escuela podemos distinguir dos categorías: la pedagógica y la arquitectónica. Desde el punto de vista pedagógico, los entornos educativos devienen más placenteros, cómodos y confortables para los educandos de infantil en aquellas zonas donde se producen:

- Uso simbólico del espacio vinculado al juego preferente.

- Actividades de aprendizaje agradable, dinámico, estimulante y poco frustrante.

- Relaciones interpersonales pacíficas.

- Estímulos sensoriales, emocionantes y cognitivos que captan su atención.

Desde el punto de vista arquitectónico, los entornos educativos devienen más placenteros, cómodos y confortables para los educandos de infantil, debido a la mayor versatilidad del espacio.

- Grado de movilidad y adecuación del mobiliario a las necesidades educativas de cada momento.

- Disponibilidad de amplitud y de áreas dilatadas para la circulación.

- Existencia de recursos e instalaciones vinculadas a las necesidades fisiológicas, así como al uso y cuidado de materiales educativos (p. 64). 
Con respecto a la acústica del aula, debe entenderse el aula como el canal por el cual se llevan a cabo procesos de comunicación. Si el canal se ve afectado por ruidos tanto internos como externos al salón de clases, el mensaje no podrá llegar de manera conveniente al receptor y, por ende, el fin educativo no se completaría. Muchos factores entran en juego al referirse al tema de la acústica, de acuerdo con Yebra, Bleda y Vera (2002):

La valoración del entorno sonoro de las aulas depende tanto de la ubicación del edificio continente (ruido medioambiental) como de los usos internos de los recintos fronterizos de las 'salas de clase' (pasillos, patios, distribuidores, servicios, vestíbulos...). Por ello aparte de la protección por distancia o por barreras arquitectónicas a las fuentes de ruido exterior clásicas, se debería tener en consideración el resto del potencial acústico contaminante que usualmente se produce en el interior del propio edificio. Y dictar normativa tendente tanto a aislar como a acondicionar dichos elementos (p.5).

En esta misma línea, Castaldi (1974) argumenta:

En la actualidad, se otorga primordial importancia a la planificación funcional de escuelas, es decir, al diseño de edificios que cumplan con las funciones para la que fueron creados en la forma más efectiva posible. Aunque se reconoce que la planificación funcional es importante, tener en cuenta las necesidades de las personas resulta igualmente importante para la planificación. Estas necesidades trascienden aquellas que se satisfacen al crear un ambiente termal, visual y acústico adecuado (p.37).

El aprendizaje debe contemplar diferentes componentes del espacio físico donde se desarrolla el proceso de aprendizaje. De acuerdo con Viñao (1993),

existen aspectos muy importantes que el arquitecto debe tener en cuenta al momento de diseñar el edificio escolar: evitar la monotonía, disminuir la construcción de las aulas sistemáticamente uniformes y eliminar de ser posible la ruptura con el ambiente familiar (bellezas naturales de los alrededores, plantaciones (p.35).

Debe entonces pensarse en la construcción de edificios educativos acogedores y promotores de deseos de permanencia en el sitio, ser un lugar donde niños y jóvenes se sientan a gusto y no deseen que la clase termine pronto. Ese sentido de pertenencia al grupo, es altamente importante. González, Marrero, Ramírez y Rodríguez (2011) indican que "el rol de la institución educativa como un grupo formal, que al igual que los grupos de pares o de amigos es capaz de fomentar en adolescentes acciones positivas las cuales lleguen a fomentar el sentido de pertenencia" (p.17). Es aquí donde también entra a jugar un rol protagónico la estética escolar para fomentar que a través de la belleza educativa el estudiante se sienta a gusto en su espacio escolar, según Errázuriz (2015)

la necesidad de mejorar la calidad estética del entorno escolar -sus condiciones materiales, espaciales, visuales, sonoras y olfativas- debería ser una prioridad de las reformas educacionales, no solamente para contribuir a generar una atmósfera más adecuada que favorezca a los procesos de enseñanza y aprendizaje, sino también para enriquecer el sentido mismo de la educación más allá de sus propósitos académicos (p.82).

En un ambiente educativo más estéticamente agradable no serán los estudiantes los únicos beneficiados, pues al lograr un clima organizacional cargado de positivismo, el personal docente se motivará aún más para realizar un trabajo con empeño, pasión y alto grado de compromiso, a la vez que los estudiantes aumentarán su rendimiento académico significativamente. Asimismo, Naranjo (2010) señala "la necesidad de contar con instalaciones físicas adecuadas y necesarias para favorecer el aprendizaje y el desarrollo de actividades académicas" (p.5). Al respecto Castro y Morales (2015) añaden que ante "la imperante necesidad de que los ambientes escolares sean estéticos, agradables, motivantes, cómodos, limpios y promuevan la estabilidad emocional que todo ser humano requiere para que el proceso de aprendizaje sea exitoso" (p.1).

Cuando se habla de ambientes de aula, debe entenderse que todo lo que forme parte de este va a influir positiva o negativamente en el proceso de enseñanza-aprendizaje, esto porque según González et ál. (2011) "las personas necesitan sentirse parte de algo, pertenecientes a un lugar o a un grupo, solo así podrán alcanzar y cubrir con éxito otros niveles de necesidades" (p. 9). Igualmente, Viñao (1993) propone que el trabajo de diseño se haga de forma completa y adecuada: 
La preocupación permanente por el buen diseño escolar garantiza que el niño esté ubicado en condiciones de percibir el mayor número de elementos que circundan la vida escolar; por ejemplo: la visualidad, iluminación, formas, textura, colores, orden, etc.; estos deben ser estimulantes de modo que, sin sobreexcitar, inciten a la actividad y no a la pasividad (p.34).

Debe entonces crearse un ambiente cómodo y positivo donde el estudiante quiera pasar casi una tercera parte del día para aprender y compartir con todos los agentes involucrados en el entorno educativo. Por su parte, continúa el autor: "el aula debe estar acondicionada para formar pequeños grupos entre los que se haga posible un intercambio simultáneo, más individual y más social” (Viñao, 1993, p.36). Debe así haber un ambiente similar al entorno social en el cual se encuentra inmersa la escuela o el colegio. González et ál. (2011) establecen la necesidad imperiosa de darle relevancia a este aspecto a fin de fomentar la permanencia en el sistema educativo:

El involucramiento tiene como resultado el éxito del estudiante en su proceso educativo, se desarrolla un compromiso fuerte y significativo que motiva a alcanzar metas a corto y largo plazo. Y, por el contrario, cuando un estudiante no se involucra en su proceso, sufre de desmotivación y desinterés que resultará en un gran riesgo de consecuencias adversas, tales como bajo rendimiento y ausentismo (p.11).

A partir de lo anterior, se analiza que el espacio escolar está lleno de significados los cuales muchas veces el educador desconoce. Puede verse cómo a nivel de educación preescolar y en algunos casos la escolar, los maestros se preocupan por decorar pegando en las paredes personajes infantiles famosos de la televisión, lo cual a nivel educativo y de aprendizaje incidental tienen un valor nulo, quizás ayuda a darle color al aula, pero no hay un significado didáctico detrás de este. Castaldi (1974) plantea la importancia de utilizar los recursos de forma creativa, el edificio por sí solo no cumple un rol educativo, deben promoverse clases dinámicas y creativas:

Las plantas escolares creativas son aquellas que utilizan de manera ingeniosa la forma, los materiales, el equipo, el color, las masas o volúmenes y el paisaje. La singularidad de diseño per se no hace que una escuela sea imaginativa, La singularidad debe contribuir al avance de la salud, la seguridad, la comodidad y la conveniencia; a la promoción de un ambiente de aprendizaje agradable, unificado, y diseñado estéticamente, y al diseño ingenioso de espacio para economía, expansión futura y fácil adaptación al cambio (p.161).

$\mathrm{Al}$ respecto Santos (1993) indica, además, una disonancia entre la educación primaria y secundaria, pues las aulas de educación infantil están bien iluminadas, adornadas con motivos diversos, tienen un cuidado material didáctico, caso contrario ocurre en las aulas de Bachillerato donde la realidad se ve reflejada en la adustez de las celdas monásticas y el deterioro causado por el uso y el descuido.

\section{Estética y calidad educativa}

La estética del aula se convierte en este factor invisible que en educación muchas veces se desconoce o no se le da la importancia merecida. "Al hablar de estética del espacio escolar debemos partir de la diversidad de elementos que determinan la misma. Abarca desde los aspectos decorativos hasta el diseño, configuración, tamaño y distribución del espacio escolar" (Delgado, 2009, p.153). Asimismo, Errázuriz (2015) indica que:

si los espacios educativos fueran estéticamente más dignos e interesantes, la experiencia cotidiana de miles de estudiantes -que deben permanecer extensas jornadas escolares en los establecimientos- tendría mayor sentido y brindaría más oportunidades para el desarrollo de la sensibilidad y de diversos modos de cognición (p. 82).

Errázuriz (2015) argumenta que no es solamente pintar paredes al inicio de cada ciclo lectivo para disimular el deterioro y hacer pensar a padres y alumnos que la escuela cumple con las condiciones físicas necesarias para nuevos procesos de enseñanza. Al decirse que el espacio debe ser un lugar estéticamente digno, el autor sugiere que sea un sitio agradable para los aprendices, que cumpla con condiciones deseables de infraestructura, accesibilidad, iluminación, ventilación, aislamiento del ruido y estética. Por otro lado, debe tomarse en cuenta que los espacios infantiles distan mucho de los requerimientos y características de los espacios para jóvenes y adultos. Cattaneo (2015) indica: “en muchas obras “modernas" la preeminencia de criterios estéticos y las indagaciones formales priman sobre la reflexión acerca de la infancia” (p.70). 
Se requiere de una cirugía estética más consistente, vale decir, reflexionar, discernir e intencionar con mayor lucidez, imaginación y pluralismo cómo los entornos escolares pueden favorecer una educación de calidad y, por ende, contribuir a un mayor desarrollo personal, social y cultural. No se trata de un problema relacionado con la decoración, sino de reflexionar cuidadosamente sobre nuestras creencias acerca de los niños, los adultos y el aprendizaje, a fin de desarrollar espacios y materiales que comuniquen un profundo respeto por los estudiantes, sus familias y el proceso de enseñanza. (Errázuriz, 2015, p.86)

La importancia de la motivación para lograr una educación de calidad es influenciada sin duda alguna por la infraestructura y estética educativa donde se desarrolla el proceso de enseñanza-aprendizaje. Cruz et ál. (2007) "lograr un clima organizacional positivo puede contribuir a aumentar la motivación tanto del personal docente como de la población estudiantil para realizar sus labores con un mayor rendimiento” (p. 24).

La realidad imperante en el sistema educativo, y que muchos jerarcas educativos pretenden pasar por alto, es la existencia de una relación directa entre la infraestructura y la calidad de enseñanza a fin de generar un impacto positivo educativo, para tal efecto factores como la comodidad, la luz, la temperatura y la calidad del aire juegan un papel muy importante. "Es importante tener presente la relación que existe entre los espacios escolares y las experiencias significativas, porque cuando le aportamos a los niños y niñas actividades a través de dicha relación se fortalecen sus conocimientos" (Palacios y Zaraza, 2013, p.29).

Existe, por tanto, una necesidad de que los espacios cuenten con un diseño rico en formas, colores imágenes y estructuras los cuales propicien la creatividad, la imaginación, capacidad de concentración y análisis de los estudiantes, donde además se estimule el juego y sobre todo donde se respire un sentimiento de comodidad. Se debe tener en cuenta que los colores producen efectos y sensaciones de manera inconsciente tal y como lo apuntan López y Gauchi (2009):

Respecto al concepto "color", la teoría del color lo define como una parte del espectro lumínico, es energía vibratoria y lo representa del siguiente modo, mediante el círculo estándar del color. Esta energía afecta de diferente forma al ser humano, dependiendo de su longitud de onda (del color en concreto) produciendo diferentes sensaciones de las que normalmente no se es consciente. (p.5).

De este modo, en lo que respecta al ambiente de aula, debe potenciarse más el uso de colores para crear sentimientos de alegría, comodidad, satisfacción entre los estudiantes. González (2015) explica: "Los colores utilizados en la guía son tonos cálidos y claros que representan alegría, pues al estar dirigida para el apoyo académico de los niños, debe presentar una composición gráfica amigable y atractiva para la retina de los pequeños" (p.58).

Es por todo lo anterior, que a la hora de seleccionar colores para pintar la infraestructura educativa, ya sea para aulas de preescolar o de educación secundaria, deben seleccionarse colores que promuevan sentimientos positivos y faciliten, por ende, el aprendizaje en cualquier nivel.

Como elemento relacionado al espacio físico cabe señalar que el color tiene una influencia muy importante en la vida del estudiantado, por cuanto los colores crean en la mente humana un efecto en la expresión de los estados emocionales, de manera que provocan diferentes respuestas que van a promover calma o excitación, frío o calor o una asociación de ideas con la alegría, la tristeza, u otros sentimientos positivos o negativos. Por ello, escoger los colores para la infraestructura de interiores debe orientarse en que la visibilidad sea fácil y natural, que el ambiente resulte confortable, que proporcione una sensación de calma, que facilite la concentración durante la clase, estimule el rendimiento y prevenga reacciones emocionales negativas (Castro y Morales, 2015, p.6).

Así las propuestas, se tiene que, al generar un ambiente estéticamente agradable, los beneficios no solamente se ven en el mantenimiento del edificio, se va más allá del valor decorativo, pues se crea un clima favorecedor al descubrimiento, en donde la enseñanza y el aprendizaje son sin duda mucho más interesantes, mejorando la calidad de vida de la comunidad escolar en general (Errázuriz, 2015). Además, debe pensarse el edificio escolar como "marco facilitador del desarrollo del proyecto educativo del centro, así como contener las configuraciones espaciales adecuadas a los modelos didácticos que vayan a ser implementados en su interior, de acuerdo al proyecto curricular de centro" (Delgado, 2009, p.44). 
Debe contemplarse entonces el valor de la estética como la presencia de un tercer maestro el cual puede influir de manera directa en la calidad educativa. Al respecto Sanz (2016) indica:

Cuando los resultados de las evaluaciones de la calidad educativa no son los deseables se dirigen las fuerzas a intervenir sobre estos factores. Sin embargo, se descuidan los aspectos contextuales que pueden interferir en todo ello. Nos referimos a factores estéticos determinantes de la calidad y el confort en la escuela, el tercer maestro (p.54).

Sin embargo, este aspecto ha sido descuidado, al punto que ha sido obviado diariamente del quehacer escolar, de acuerdo con Errázuriz (2015),

La invisibilidad del tercer maestro no es de extrañar. Prueba de ello es que, generalmente, los discursos y práctica referidos a la educación omiten y/o desconocen la importancia del entorno educativo, lo cual se refleja en la ausencia de investigación y de políticas públicas que contribuyan a mejorar las condiciones físicas, materiales y estéticas del ambiente escolar (p. 95).

Es entonces donde debe valorarse esta situación, a fin de tener un sentido mucho más integral de los procesos educativos y poder así lograr la calidad de los mismos. Nunca serán neutros elementos tales como el color, la luz, los sonidos, áreas verdes, todos ellos juegan un rol de suma importancia y deben pensarse con responsabilidad, pues estos serán parte crucial en los procesos de aprendizaje de los niños y jóvenes del sistema educativo. Cañizares, citado por Errázuriz (2015):

(...) plantea que la interacción con el entorno es fundamental al considerar el diseño de un edificio destinado a la educación. Desde esta perspectiva, incorporar a su arquitectura rasgos propios de la cultura local puede facilitar la creación de un sentido de pertenencia a la comunidad educativa (p.96).

$\mathrm{Al}$ respecto Cruz et ál. (2007) consideran que es importante tomar en cuenta aspectos externos al entorno educativo, es decir aquellos que identifican al contexto donde se encuentra ubicada la institución. Todo esto con el fin de mejorar relaciones entre la escuela y la comunidad y entre la escuela y las familias de la población estudiantil, lo cual sin duda alguna va a mejorar la calidad educativa de manera indirecta. Delgado (2009) indica que "si pretendemos que la arquitectura escolar responda a las necesidades educativas de cada comunidad, es necesario que exista una interrelación entre los criterios arquitectónicos (estéticos, funcionales, constructivos y ambientales), los criterios pedagógicos y los criterios sociales" (p.48). De igual forma, Palacios y Zaraza (2013) apuntan:

La escuela en relación con los procesos artísticos fomenta el intercambio y se convierte en el lugar propicio para exteriorizar
las ideas por medio de materiales, posibilitando las discusiones, las conversaciones y de esta manera favorecer la integración
social, debido a esto se creó una conciencia crítica en los alumnos, donde la comunicación visual constituye un elemento
fundamental en el proceso de desarrollo del niño individual y colectivo que le permite liberarse mediante la creación y la
expresión por medio de la incorporación a nuevas técnicas artísticas, se presenta un amplio campo de posibilidades expresivas
que posibilita que el niño sea capaz de conservar, expresar y producir sus propias experiencias y además que los estudiantes
desarrollen su capacidad de crear y reelaborar nuevos elementos a partir de las experiencias y con nuevos planteamientos
(p.26).

\section{Deterioro de la infraestructura educativa}

Un deterioro de la estructura puede deberse a múltiples factores, para Padilla (1998),

La mayoría de los usuarios de la planta física expresan que el deterioro de sus centros educativos se debe a la falta de recursos económicos para realizar acciones de conservación y mantenimiento, lo que no es totalmente cierto, ya que otros planteles mantienen en buen funcionamiento sus centros con pocos recursos (p.3).

Es entonces donde el papel del administrador educativo juega un papel importante para velar por el mantenimiento del inmueble a su cargo. El deterioro del edificio escolar se puede apreciar tanto a nivel de infraestructura como en el mobiliario, el déficit de los pupitres y el material didáctico muchas veces va en oposición con los objetivos de la calidad de la educación. 
De acuerdo con Padilla (1998) los daños pueden clasificarse en daños mayores, menores, daños estructurales, daños no estructurales, daños químicos, y combinaciones de los anteriores. A nivel de costos, los daños menores deben atribuírsele un $15 \%$ del valor total de los elementos, por el contrario, a los daños mayores debe atribuírsele el $60 \%$ de los costos, además debe contemplarse que su reparación debe interrumpir las actividades académicas-administrativas. Cajiao (2012) define los tipos de daños de la siguiente forma:

Obras de Mantenimiento Menor, son todos aquellos trabajos o actividades que deben realizarse para rehabilitar o corregir el deterioro o fallas detectadas en los inmuebles en sus instalaciones; es decir, obras que por sus características se pueden realizar de forma satisfactoria con la participación de un obrero especializado.

Obras de Mantenimiento Mayor, son todos aquellos trabajos o actividades que deben realizarse para rehabilitar o corregir el deterioro o fallas detectadas en los inmuebles o instalaciones, es decir obras que por sus características técnicas afectan la estructura del edificio y que por su dificultad, especialización y riesgo no se pueden realizar únicamente con la participación de un obrero especializado si no que necesita la intervención y el respaldo de un profesional en las áreas de la arquitectura, la ingeniería civil o ingeniería eléctrica (p. 65).

\section{Deserción escolar y su relación con los daños de la infraestructura}

Muchas veces se atribuye el abandono escolar a factores psicológicos, sociales y económicos que vive la comunidad estudiantil, pero muy pocas veces se analiza dicha causa a factores relacionados con el deterioro de la infraestructura educativa. Álvarez et ál. (2006) definen la deserción como:

Una manifestación de conducta que podría ser el resultado de la interacción de una serie de variables, reflejada en la decisión, por parte de la persona, de abandonar sus actividades académicas; cabe destacar que esta decisión que se toma es producto de situaciones que en muchos casos van forzando a los estudiantes y las estudiantes a no volver al colegio, sin que medie un análisis cuidadoso de las implicaciones de esa decisión, ni se hayan buscado alternativas de solución a los problemas (p.10).

Debe revisarse a la vez el concepto de calidad educativa, pues los autores analizan también que, en instituciones con altos índices de calidad educativa se deben tener valores mínimos de deserción escolar. Partida (2005) citado por Álvarez et ál. (2006) mantiene:

No es posible que habiendo calidad en la enseñanza haya estudiantes que desertan, puesto que la calidad implica entrega y conocimiento por parte del educador y la educadora, la buena comunicación con sus estudiantes, la satisfacción de las necesidades propias del estudiantado, el esfuerzo para hacer que descubran la gran capacidad que tienen y muchas otras características que en la realidad no están presentes cuando se da la deserción (p.12).

Si bien es cierto, los gobiernos hacen grandes esfuerzos para becar estudiantes de escasos recursos a fin de que no abandonen las aulas, muchas veces este esfuerzo no es suficiente para lograr que los estudiantes continúen dentro del sistema educativo. El estado de deterioro de los edificios, el abandono que se vive dentro de las aulas desmotiva al estudiante y esto provoca aún más el desinterés por continuar con sus estudios.

Si bien es cierto, son varios los factores que influyen en la deserción Álvarez et ál. (2006) los desglosa de la siguiente manera:

Una dosis considerable de decisión de abandonar el colegio por la atracción de otras alternativas vinculadas al mundo del trabajo, pero también al consumo.

La incapacidad del propio sistema escolar de acoger y dar respuesta a jóvenes y a sus necesidades educativas. Los programas de educación están obsoletos, algunos no responden a las expectativas estudiantiles, ni a la sociedad, ni siquiera al nivel universitario.

Inadecuación de la educación media en relación con las formas educativas, las prácticas pedagógicas, la forma de convivencia, normatividad y disciplina escolar, la atención a la diversidad y otros, que no asumen la realidad vital y la especificidad cultural de las poblaciones juveniles.

Docentes que no estimulan la integración cultural, sino que generan una separación que excluye a poblaciones de inmigrantes, lo que provoca abandono de las aulas. 
Falta de una adecuada pedagógica, de técnicas participativas que hagan la educación más atractiva para las poblaciones jóvenes de la sociedad actual, falta de dominio en el aula por parte del educador que promueve el descontento, la indisciplina y el abandono del sistema educativo (p.14).

Ante la ausencia de motivación por parte de los docentes, la carencia de material didáctico y los pocos espacios de esparcimiento que existen en las instituciones educativas, la infraestructura ejerce un papel preponderante ante dicha problemática.

\section{Metodología}

La investigación corresponde a un estudio mixto porque se utilizaron técnicas tanto cualitativas como cuantitativas para la recolección y análisis de resultados.

\section{Población}

La población analizada en la Región Pacifico Central muestra grandes índices de pobreza y pobreza extrema, por lo cual se logró comprobar que en los centros educativos donde se realizó el estudio, brindan apoyo a sus estudiantes por medio de programas de beca.

El estudio se llevó a cabo en treinta y tres instituciones educativas las cuales son parte de la Región Pacífico Central de Costa Rica. Al tratarse de un estudio regional, el área seleccionada corresponde a los cantones que atiende la Sede del Pacífico de la Universidad de Costa Rica, a través de sus proyectos de Investigación y Acción Social.

Colegios: como parte de los colegios involucrados se encuentran el Liceo José Martí, Liceo de Chacarita, Liceo Antonio Obando Chan, Colegio Técnico Profesional de Puntarenas, Liceo de Miramar, Liceo Diurno de Esparza, Liceo Rural de Tárcoles, Colegio Técnico Profesional de Quepos, Colegio Técnico Profesional de Parrita, Emiliano Odio, Colegio Técnico Profesional de San Mateo, Liceo Quebrada Ganado, Colegio Técnico Profesional Jaco y Colegio Técnico Profesional de Orotina.

Escuelas: dentro de las escuelas analizadas se encuentran la Escuela El Carmen, Delia Urbina de Guevara, Mora y Cañas, El Roble, Riojalandia, José María Zeledón, Quebrada Ganado, Tárcoles, La Hacienda Jaco, Escuela Jaco, La Julieta, República de Corea, Tobías Guzmán, Primo Vargas Valverde, El Barón, Arturo Torres, Marañonal, Heriberto Zeledón Rodríguez y Rogelio Sotela Bonilla.

Cabe señalar que casi la mitad de estas instituciones atienden entre 200 y 500 estudiantes, lo cual quiere decir que son edificaciones las cuales albergan gran cantidad de niños y jóvenes. Es importante mencionar, además, que estas son comunidades que muestran grandes índices de pobreza y pobreza extrema. Lo cual se puede observar porque un $54 \%$ de las instituciones cuenta con una población que vive con insuficiencia de recursos económicos o bajo la influencia de problemas económicos, pues perciben un ingreso aproximado de 100 mil colones por mes, según encuesta realizada. El otro 23\% de las instituciones posee estudiantes que no pueden suplir las necesidades básicas para sobrevivir, por lo que entran en la categoría de pobreza extrema, recibiendo de 30 a 40 mil colones mensuales. El 15\% de las entidades cuenta con una población que vive bajo una economía regular; y tan solo un $8 \%$ de la población posee la capacidad para satisfacer sus necesidades.

Como respuesta a la situación descrita en el párrafo anterior, se logró comprobar que los centros educativos donde se realizó el estudio, brindan apoyo a sus estudiantes por medio de programas de beca, ya que en un 92\% se benefician de los distintos programas de becas que existen y tan solo en una de las instituciones no se ofrece tal beneficio o no cuenta con las posibilidades de brindar tal ayuda a sus estudiantes. 


\section{Instrumentos}

Para recabar la información requerida se diseñaron dos tipos de instrumentos, uno fue una tabla de cotejo para guiar la observación de campo realizada en cada uno de los centros escolares y el segundo fue un cuestionario dirigido tanto a estudiantes como a docentes y administradores educativos.

La guía de observación contemplaba aspectos a evaluar durante la visita, tales como los relacionados con paredes, cielorrasos, ventanas, estructura del techo, desfogue pluvial, dimensionamiento de pasillos y puertas, instalaciones sanitarias, instalaciones eléctricas, mobiliario, iluminación exterior, basureros, zonas verdes y vegetación, áreas recreativas, comedor, manejo de desechos, riesgos ambientales, extintores, hidrantes, entre los aspectos más relevantes relacionados con la estructura, instalaciones mecánicas, mobiliario, accesibilidad, servicios complementarios, entorno, seguridad y salud. Se evaluó cada uno de los aspectos utilizando una escala de malo, regular, bueno, muy bueno y excelente. La información recolectada también fue respaldada a través del recurso fotográfico.

El cuestionario aplicado a estudiantes y docentes estaba conformado por dos preguntas abiertas y ocho preguntas cerradas a fin de conocer con precisión la opinión de ellos con respecto a la estética del aula y de la institución educativa en general para valorar el grado de influencia que ejerce el tercer maestro en la calidad de la enseñanza.

Dado que el enfoque del estudio fue de carácter mixto, el análisis de los resultados se elaboró analizando los datos tanto cualitativos como cuantitativos. En dicho instrumento se analizan los elementos y factores involucrados en la calidad del ambiente escolar relacionados con las dimensiones del aula, calidad del mobiliario, nivel de ruido, orden y aseo del aula y del entorno escolar, materiales didácticos, colores e iluminación del espacio escolar.

\section{Resultados}

\section{Condiciones de infraestructura}

El estado actual de la infraestructura en educación primaria y secundaria en la Región Pacífico Central destaca por el hecho de que muchas de las instituciones observadas están remodeladas y nuevas, otras en proceso de remodelación, otras por el contrario están en muy malas condiciones e inclusive algunas instituciones alquilan las instalaciones porque no tienen un inmueble propio para impartir las lecciones. Es claramente apreciable que no se le ha dado la importancia a este tercer maestro dentro del sistema educativo de la región bajo estudio. Al respecto, Gutiérrez (1998) coincide en que:

La configuración de los centros no debe pasar inadvertida a la labor del docente, ya que, tanto si se desea como si no, el espacio emite mensajes de manera continua que penetran en los alumnos, sea cual sea el tipo de programa o las expectativas del profesor (p.76).

Es lamentable ver el descuido general causado por el abandono del inmueble, las canoas en mal estado, paredes de madera con huecos, aulas con dimensiones pequeñas para grupos tan grandes, techos que necesitan un cambio por la gran cantidad de goteras, cabe anotar, además, que en varias instituciones no se utiliza vidrio en las aulas para la ventanería, sino que se utiliza adoquín, malla o verjas porque son instituciones ubicadas cerca de áreas costeras y, por lo tanto, se experimentan altas temperaturas durante el día. Sin embargo, lo anterior hace que los estudiantes se vean expuestos a altos niveles de ruidos y se distraigan con mayor facilidad durante las lecciones, provocando a la vez problemas de disciplina dentro de las aulas.

Al tomar en cuenta los aspectos relacionados con la estética escolar, es lamentable ver cómo aún hay mucho por corregir. Sobre esto, Gutiérrez (1998) apunta que 
La realidad escolar que hoy seguimos encontrando continúa presentando las mismas características de rigidez, monotonía y pobreza que en años anteriores. Salvo raras excepciones de algunos colegios (generalmente privados), los centros carecen de espacios abiertos con vegetación, del equipamiento sanitario para el aseo tras el desarrollo de actividades de educación física, de lugares propicios para la labor artística (p. 214).

Esta situación se ve evidenciada dado que un alto porcentaje de la población estudiantil entrevistada indica que los niveles de ruido percibidos son muy altos. Mencionan, además, que durante los recreos o tiempos de comida, la bulla es aún mayor porque los estudiantes en receso gritan demasiado. Es importante tomar en cuenta que debe hacerse un esfuerzo para reducir los niveles de ruido, pues todo el ambiente escolar se ve afectado, el problema se incrementa producto del diseño de ventanas abiertas que impiden que el ruido se concentre en un solo lugar; esto afecta la salud mental tanto de estudiantes como de docentes, ya que al comunicarse a través de gritos, emergen serios problemas de disciplina y de concentración para un aprendizaje de calidad.

En relación con el mobiliario, en muy pocas de las instituciones analizadas, especialmente en escuelas primarias, los estudiantes consideran que el mobiliario es apropiado y se encuentra en buenas condiciones, sin embargo, en los colegios más de la mitad de la población entrevistada indica que el estado de los pupitres, y mobiliario en general dentro del aula, no es agradable. Dentro de los daños indican que están muy sucios, no son cómodos y están en malas condiciones generales.

El estudio también ha tomado en cuenta durante las observaciones realizadas el mobiliario urbano, el cual contempla lo siguiente: bancas, iluminación exterior, basureros, y mesas. En las diversas instituciones que se analizaron, se logró observar que mayoritariamente todas poseen estos mobiliarios dentro de las instituciones; unos muy coloridos y atractivos en comparación con otros en esta misma región los cuales necesitan ser reemplazados y estéticamente no son muy atractivos, pues cuentan con un estado de descuido muy notorio. Por lo tanto, se puede aducir que muchas instituciones deben implementar cambios, pues el estado regular es apenas apto para el desempeño educativo.

Por otro lado, el aula como tal, en ocasiones alberga hasta cuarenta personas menores o jóvenes en el mismo espacio; en un clima cálido como el de la Región Pacífico Central ocasiona problemas de indisposición o sentido de pertenencia a un espacio específico, pues de forma inconsciente se genera un rechazo y se desea salir de ahí. Sin embargo, cabe señalar que las instituciones analizadas, al menos en un $75 \%$ de ellas, se han realizado muchos esfuerzos para reducir la cantidad de estudiantes por grupo y esto se ve reflejado en las respuestas que dan los educandos, la gran mayoría indica que cuenta con espacio suficiente para movilizarse dentro de su salón de clases. Según los lineamientos establecidos en las Normas de Infraestructura Física Educativa del Ministerio de Educación Pública, debe existir un área de $1.5 \mathrm{~m} 2$ por estudiante, esto con el fin de promover que los educandos estén en constante movimiento, dinamizando así el proceso de aprendizaje. Sin embargo, muchas veces, aunque se tenga este espacio no se utiliza para romper los paradigmas de lo que es una clase tradicional tal y como lo apunta Viñao (1993) "pero lo usual, por el momento, es la resistencia a introducir innovaciones estructurales en los programas docentes que solo sirven para dar una visión superficial de los mismos sin poner en entredicho el esquema tradicional" (p.14).

Los centros educativos públicos manejan el presupuesto a través de las Juntas de Educación. En la mayoría de los casos, especialmente en zonas rurales, los rubros que se incluyen no contemplan partidas ni fondos suficientes para la construcción de aulas, ni espacios escolares, sino más bien para reparaciones y en algunos casos ampliaciones o mejoras. Lo cual se convierte en una debilidad, ya que ante los daños que sufre la infraestructura educativa no es posible mejorar las condiciones.

Sevilla, Sanabria y Shedden (2010) indican, además, que la infraestructura debe cumplir y sujetarse a normas con respecto al color y los materiales que se utilicen a lo interno de las aulas:

Los colores internos de las aulas, laboratorios y talleres deben ser tonos claros para contribuir con la iluminación, debido a que existe mejor distribución de la luz cuando incide sobre las superficies. Algunas recomendaciones son: 
- Cielos: preferiblemente deben ser color blanco mate o con un factor de reflexión de 75\%, este factor permite reflejar la luz de manera difusa, disipando la oscuridad y reduciendo los brillos de otras superficies. Esto implica un ahorro en la iluminación artificial.

- Paredes y pisos: las superficies de las paredes situadas a nivel de los ojos pueden provocar deslumbramiento. Los colores pálidos con factores de reflexión del 50 al $75 \%$ suelen ser adecuados para las paredes. Aunque las pinturas brillantes tienden a durar más tiempo que los colores mate, son más reflectantes. Por consiguiente, las paredes deberán tener un acabado mate o semibrillante. Los acabados de los pisos deberán ser de colores ligeramente más oscuros que las paredes y los techos para evitar brillos. El factor de reflexión de los suelos debe oscilar entre el 20 y el $25 \%$ (p.52).

Por otro lado, con respecto a las instalaciones mecánicas, se toman en cuenta los siguientes aspectos: instalaciones sanitarias, baños para personas discapacitadas, instalaciones eléctricas y lavamanos. Generalmente lo que se destaca sobre estas instalaciones son los malos olores y en algunos casos son completamente insalubres. Por otro lado, otro aspecto observado fue el cerramiento, el cual es uno de los aspectos más importantes para la observación que lleva a cabo este proyecto debido a que de él depende la seguridad de los y las estudiantes de la Región Pacífico Central. Una buena seguridad que tome en cuenta un amplio y buen estado del cerramiento de la institución, podría evitarles muchos dolores de cabeza a los padres y encargado de estas diversas instituciones de educación pública. En la observación se pudo ver que muchas instituciones no cuentan con un cerramiento por completo, sino en solo una parte del terreno donde los niños o cualquier otra persona externa a la institución puede entrar o salir sin mayor dificultad. Sin embargo, cabe destacar que muchas de las instituciones cuentan con mallas nuevas al igual que tapias de baldosas o cemento, otras con cerramiento en vegetación. De esta manera, se puede tener una visión sobre la inseguridad y riesgos que puedan tener los estudiantes dentro de estas escuelas y colegios públicos de la Región Pacífico Central del país.

Otro aspecto que no debe dejarse de lado al estudiar las condiciones de infraestructura educativa es el relacionado con la accesibilidad, cada institución visitada ha sido muy diferente a las otras; se han observado aspectos relacionados con el acceso al parqueo, parqueo para discapacitados, rampas de acceso, pasos cubiertos, superficie de rodamiento, entrada principal, entrada secundaria, demarcación vial interna. Los aspectos anteriormente mencionados son importantes para así cumplir con lo estipulado mediante la Ley 7600. El resultado de la observación realizada muestra que muy pocas instituciones cuentan con rampas, parqueos y parqueos para personas discapacitadas, lo cual deja ver cómo el mismo edificio escolar está excluyendo a personas que puedan tener algún tipo de discapacidad y pudieren necesitar de condiciones especiales de acceso al centro educativo.

En cada una de las instituciones educativas también se evaluaron los servicios complementarios, de los cuales se tomaron aspectos tales como: áreas recreativas, comedor, enfermería, biblioteca, manejo de desechos y fotocopiadora, que cada institución tiene como parte de su apoyo institucional para brindar los recursos complementarios que los estudiantes necesitan para un desarrollo óptimo y esencial de su aprendizaje y desarrollo físico e integral dentro del sistema educativo. La observación determinó que muchas instituciones cumplen en su mayoría con los servicios complementarios de manera óptima y que otras instituciones por el contrario carecen de estos. Sin embargo, solo dos de las instituciones cuentan con enfermería, las otras solo tienen sus propios botiquines y reciben visitas de funcionarios de la Caja Costarricense del Seguro Social (CCSS) los cuales vacunan a los niños y adolescentes.

La institución educativa está siempre inserta en un entorno el cual tiene características muy particulares, las cuales deben siempre tomarse en cuenta para que exista una armonía con el sistema educativo. En la investigación se consideraron aspectos relacionados con el parqueo de bicicletas, superficie en césped, superficie sellada, superficie tierra/lastre, arborización y jardines, accesibilidad a transporte público, riesgos ambientales, demarcación horizontal y vertical. Dichos aspectos mencionados anteriormente son de gran 
importancia para la población estudiantil dado que estos van de la mano con la seguridad que muchos estudiantes puedan gozar y, por ende, van a tener implicaciones en el desarrollo educativo de cada región.

\section{Estética educativa}

La percepción de estudiantes tanto de primaria como de secundaria con respecto al tamaño del aula es apropiada, según sus opiniones. Se logró constatar que efectivamente los estudiantes tienen espacio suficiente para circular, pero lastimosamente los pupitres son colocados de manera tradicional, en filas viendo hacia la pizarra, durante las observaciones se logró constatar que en ninguna de las instituciones se varía la posición para hacer trabajos en grupo ni dinámicas. Ante esta lamentable situación, Delgado (2009) afirma que "en definitiva, deben tenerse en cuenta tanto la dimensión psicológica como la dimensión estética del espacio escolar. ya que ambas pueden repercutir positiva o negativamente en la práctica docente y el ambiente de aprendizaje de las instituciones educativas" (p.155).

Sobre el mismo tema relacionado con el aula escolar, se procedió a preguntar a los estudiantes por los materiales empleados durante el proceso de enseñanza y aprendizaje. Al respecto, al menos la mitad de los entrevistados considera que los materiales son apropiados y atractivos para llamar su atención. Sin embargo, un porcentaje significativo de los entrevistados indica que los materiales son utilizados esporádicamente y algunos opinaron que son muy viejos y no les atraen. Durante las observaciones y entrevistas con docentes se constata que los materiales didácticos los tienen guardados en bolsas plásticas para evitar el deterioro y así poderlos seguir utilizando con otros grupos. Vemos como los espacios escolares, lejos de potenciar el aprendizaje incidental a través de la observación de material didáctico colgado en los salones de clase, más bien son espacios vacíos o con decoraciones o dibujos de personajes de la televisión sin ningún mensaje ni finalidad didáctica.

En las instituciones observadas se evidenció, además, la disponibilidad con la que cuentan para la utilización de tecnología dentro del aula; en el $92 \%$ de los casos, las instituciones contaban con la posibilidad de utilizar recursos tecnológicos. Un $8 \%$ de las instituciones; es decir, tan solo una institución no contaba con recursos tecnológicos.

Es necesario que dentro de las aulas exista disciplina, orden y aseo. Al consultárseles al respecto, los estudiantes piden que las aulas sean más ordenadas, mejor decoradas, con colores llamativos, más limpias y con iluminación y ventilación convenientes. Al observar se logra comprobar dicha necesidad descrita por los entrevistados, pues las paredes, cielorrasos, puertas, mobiliario y pizarras muestran un deterioro notable, el descuido es grande. Esta situación viene a repercutir tanto en el ánimo del docente para impartir la clase, como la disposición y motivación del estudiante para adquirir nuevos conocimientos. Es claro entonces que algo está fallando, ya que de acuerdo con Cajiao (2012) al Ministerio de Educación Pública:

(...) le corresponde promover el desarrollo y consolidación de un sistema educativo de excelencia que permita el acceso de toda la población a una educación de calidad, centrada en el desarrollo integral de las personas y en la promoción de una sociedad costarricense integrada por las oportunidades y equidad social (p. 58).

Este dato evidencia que hay mucho por mejorar, y llama mucho la atención como los mismos estudiantes, ante la entrevista realizada sobre estética e infraestructura educativa, hacen un llamado para que las clases se vuelvan más dinámicas, que haya más diversión y sugieren el aprendizaje a través de juegos para lograr una metodología mucho más atractiva y con mejores resultados. 


\section{Implicaciones de la infraestructura y la estética educativa en las condiciones estudiantiles y docentes}

$\mathrm{Al}$ analizar tanto las condiciones de infraestructura educativa, así como la estética dentro del aula, vemos como ambos factores influyen de forma directa en el desempeño tanto docente como del estudiante. Naranjo (2010) plantea que deben existir instalaciones físicas adecuadas y condiciones necesarias para que exista un verdadero aprendizaje. Se necesita que el ambiente escolar motive tanto a todos los actores del proceso educativo y que no excluya o expulse a nadie del mismo.

Lo anterior va relacionado directamente con el rendimiento académico de los estudiantes. Se observa que, del total de las instituciones observadas, un 35\%, de las instituciones asegura que sus estudiantes tienen una gran dificultad en la asignatura de Matemáticas. Un 27\% en Estudios Sociales. Asimismo, se logró visualizar que un 7\% de las instituciones presenta dificultad en inglés; mientras que un 4\% en la asignatura de Química. Al existir estas debilidades, alguna acción debería tomarse en cuenta para colaborar y reducir estos porcentajes de deserción. Los colegiales, por su edad y etapa en que se encuentran, ya enfrentan múltiples conflictos relacionados con la adolescencia, tal y como lo afirman González et ál. (2011):

Como en otros momentos de la vida, las personas en la etapa de la adolescencia se enfrentan a un nuevo mundo, lleno de ambientes por conocer, muchas personas nuevas, situaciones tan diversas y además todos los cambios fisiológicos y psicológicos que ya se conocen, propios de la adolescencia (p.10).

Debe entonces la institución educativa dar apoyo y no obstaculizar procesos educativos a través de la creación de ambientes más interesantes y dinámicos.

Como parte de los principales desafíos de la educación se logró comprobar que los docentes de la Región Pacífico Central requieren capacitación en diversas ramas, especialmente sobre aspectos relacionados con la didáctica, se requiere mayor capacitación en el área de evaluación, pedagogía, confección y uso de materiales didácticos. Esto porque se observó que las clases se basan únicamente en el dictado o escritura de resúmenes sobre los temas bajo estudio. Llama la atención la necesidad descrita acá dado que los docentes que trabajan en las instituciones de la región en estudio, cuentan con estudios de educación superior. Del total de los educadores, un $38 \%$ posee licenciatura. Un $34 \%$ de ellos goza del título de maestría; y un $28 \%$ solo tiene bachillerato universitario, por lo que la necesidad surge no por falta de preparación académica, queda aquí la interrogante si más bien esta situación se debe a la desmotivación que se emite del descuido de la estética y la infraestructura educativa.

Cuando se le consulta al personal docente sobre el sentido de pertenencia, un $85 \%$ de ellos afirma ser alto, que existe pertenencia y permanencia en la institución y se sienten parte de ella, solo un $15 \%$ indica que no se sienten parte de la institución en la que laboran. Lo cual, lamentablemente repercute directamente en la población estudiantil.

\section{Conclusiones}

Como parte de este proceso investigativo, es posible llegar a diferentes conclusiones con respecto al tema analizado. Para empezar, es notorio como en la distribución de los espacios de aula se destaca la prevalencia de la distribución de los alumnos en pupitres individuales, no se favorece el aprendizaje activo y cooperativo. A pesar de que haya suficiente espacio para mover el mobiliario, este permanece estático y solamente se visualiza una vía de comunicación: de profesor a alumno.

En relación con la dotación de material didáctico y recursos tecnológicos, cabe destacar que en la gran mayoría de los centros escolares analizados cuentan con disposición de utilizarlos, el problema radica en que el docente no los usa por desconocimiento o para que no se deterioren. Es claro apreciar también que las 
aulas cuentan con una decoración muy limitada, desplegándose material de dibujos en centros educativos infantiles para capturar la atención de los niños, pero el material en sí carece de mensajes educativos.

Debido a las condiciones climáticas de la zona, la infraestructura se trata de adaptar para tener aulas más frescas, con paredes que no llegan hasta el techo para facilitar la ventilación, sin embargo, la acústica se ve muy afectada porque el ruido de todas las aulas se mezcla ocasionando un ambiente muy incómodo y difícil para poder impartir lecciones. Se logró apreciar cómo el docente tiene que forzar mucho la voz para poder dar indicaciones a sus estudiantes y estos a su vez se ven involucrados en problemas de disciplina porque no logran concentrarse debido al exceso de bulla que se percibe.

Los salones de clase cuentan con buena iluminación pese a que en muchos inmuebles se presentan problemas con la conexión eléctrica o bien porque hay mucho descuido y los bombillos están quemados, por lo que un punto a favor es que las clases se llevan a cabo durante el día y no se depende de la electricidad para contar con buena iluminación.

El tamaño tanto general del inmueble como de los salones de clases es adecuado, en su mayoría cuentan con zonas de esparcimiento o de recreo para que los estudiantes puedan disfrutar durante los recesos. Sin embargo, es una lástima apreciar cómo a pesar de que se cuenta con el tamaño necesario en zonas verdes, muchas veces está demasiado alto el césped y los estudiantes no pueden tener acceso para jugar. De igual manera sucede en las aulas, aunque se tenga el espacio adecuado para realizar dinámicas grupales, el docente se limita únicamente a dictar o escribir en la pizarra, anulando cualquier intento de trabajo cooperativo que se quiera desarrollar.

Se debe reconocer que el color de las escuelas y colegios se vislumbra como un punto a favor dentro del análisis realizado, porque a pesar de haber descuido en otros campos de la infraestructura y la estética, la mayoría de las autoridades educativas se preocupan por pintar periódicamente las paredes de los centros educativos. Cabe mencionar, además, que quizás los colores utilizados no son los más apropiados porque se ha logrado comprobar que el color siempre ejerce una relación directa en el estado de ánimo. Quizás los colores no están fomentando la creatividad ni motivando a tener espacios con mayor motivación dentro de las aulas. Es acá entonces donde lo que estaría faltando por mejorar es la adecuada selección de colores para propiciar la construcción de aulas de escuela y colegios más productivas.

Por otro lado, es lastimoso ver cómo el descuido llega a tal punto en que el aseo se ve afectado provocando condiciones inadecuadas para llevar a cabo cualquier labor, y mucho menos una labor educativa. Los pasillos, las aulas, los servicios sanitarios y las zonas de recreo son las principales víctimas del desaseo, el mobiliario y las paredes se encuentran sumamente rayados y con mensajes donde se denota gran cantidad de antivalores presentes.

Por lo tanto, contamos con un sistema educativo que ha descuidado abruptamente las instalaciones físicas y ha dejado a la estética relegada a un segundo plano. No se le ha dado la importancia a este tercer maestro que habla por sí solo, capaz de promover el aprendizaje de manera autónoma y motivar para que los verdaderos procesos educativos se lleven a cabo de manera significativa. Se ha visto entonces cómo el entorno y los espacios escolares influyen de forma directa, positiva o negativamente en los procesos de enseñanza. En el caso de la Región Pacífico Central, el estudio demuestra cómo a pesar de contar con las dimensiones de terreno y de infraestructura educativa, no se aprovecha el espacio fomentando sentimientos de pertenencia y permanencia en la institución, el espacio por sí solo excluye y desmotiva al estudiantado por lo que se aumentan los índices de deserción y bajo rendimiento académico.

De este modo, a manera de recomendación se considera sumamente necesario la implementación de algunos cambios tanto por parte de la administración de la institución educativa como por parte de los docentes de grupo. Entre ellos se destaca:

- Es necesario capacitar al docente para poder utilizar herramientas tecnológicas que le permitan al estudiante involucrarse más en la dinámica de la clase a fin de aprovechar tantos los recursos físicos como didácticos con que cuenta el centro educativo. 
- Promover ambientes lúdicos dentro del aula, evitar esa transmisión unidireccional de conocimientos docente-estudiante. Mover los pupitres, sentarse en el suelo, hacer círculos de conversación, jugar y divertirse para aprender de manera significativa con los alumnos.

- Los docentes de centros educativos cuya infraestructura no ayuda a tener ambientes limpios acústicamente, deben manejar estrategias metodológicas y disciplinarias que les permitan ayudar a los estudiantes a moderar el volumen de su voz al hablar.

- Por último, muchas veces lo que se aprende fuera de las aulas es más enriquecedor que lo que se estudia en una pizarra o en un libro, por lo que se motiva al educador a utilizar las zonas verdes, canchas, y otros espacios dentro de la infraestructura educativa para desarrollar parte de sus clases no solo en el salón de clases.

Finalmente, cabe anotar como conclusión del análisis realizado que el educador y administrador educativo se preocupan muchas veces por cumplir con tener doscientos días lectivos, pero desconocen que la cantidad de tiempo no es sinónimo de calidad educativa, pues con espacios físicos deteriorados o descuidados, donde hay demasiado ruido y olores desagradables, donde los materiales didácticos se vean reducidos a contar con posters de personajes de la televisión, es muy difícil que un estudiante llegue motivado cada día al salón de clases; y el problema es no solo la desmotivación estudiantil, sino también los mismos docentes que dejan de capacitarse y de hacer un esfuerzo cada vez mayor ante los retos del trabajo con niños y adolescentes para llevarles estrategias didácticas innovadoras y darle un mayor dinamismo al sistema educativo en general.

\section{REFERENCIAS}

Álvarez, M. W., Arias, D. A., Cortes, M. C., Montiel, O. E., Rosales, F. X. y Umaña, V. E. (2006). Factores asociados a la deserción de la población estudiantil de sétimo año del Liceo José Martí, del Liceo de Chacarita y del Liceo Antonio Obando Chan, en el año 2004. (Tesis de Licenciatura). Universidad de Costa Rica, Costa Rica.

Cajiao, A. (2012). Parámetro de diseño para escuelas sostenibles en Costa Rica. (Tesis de Licenciatura). Universidad de Costa Rica, Costa Rica.

Castaldi, B. (1974). Diseño de centros educativos. México: Editorial Pax México.

Castro, P. M., y Morales, R. M. (2015). Los ambientes de aula que promueven el aprendizaje, desde la perspectiva de los niños y niñas escolares. Revista Electrónica Educare, 19(3). doi: http://dx.doi.org/10.15359/ree

Cattaneo, D. (2015). Arquitectura escolar moderna: interferencias, representación y pedagogía. Voces y Silencios: Revista Latinoamericana de Educación, 6(1). Recuperado de https://bit.ly/2G29UiY

Cruz, V. M., Jiménez, B. L., Jiménez, L. E., Méndez, D. Y., Vivas, S. A., y Zamora, A. W. (2007). Factores que favorecen el desarrollo de una actitud positiva hacia la docencia. (Tesis de Licenciatura). Universidad de Costa Rica, Costa Rica.

Cordero, M. (2 de octubre de 2018). Escuelas y Colegios con pésima infraestructura pese a disponer de $\$ 150$ mil millones. Semanario Universidad. Recuperado de: https://bit.ly/2RCHBsO

Delgado, V. (2009). Organización del aula de Educación Primaria en centros educativos de Burgos y su provincia. (Tesis de Licenciatura). Universidad de Burgos. Recuperado de Repositorio Institucional de la Universidad de Burgos.

Errázuriz, L. (2015). Calidad Estética del Entorno Escolar: El (f)actor Invisible. Arte, Individuo y Sociedad, 27(1). Recuperado de https://bit.ly/2KYZ8IW

Fallas, C. (10 de enero de 2016). Bancos invierten más para tener operaciones sostenibles. El Financiero. Recuperado de https://bit.ly/2FZ1AQQ

Fernández, A. (1997). La Expresión Arquitectónica en los Edificios Escolares. Recuperado de https://bit.ly/2rmJcr9 
González, P. Z., Marrero, T. D., Ramírez, G. M., y Rodríguez, M. M. (2011). Relación entre el sentido de pertenencia y el éxito académico en estudiantes de educación media, un aporte desde la Orientación. (Tesis de Licenciatura). Universidad de Costa Rica, Costa Rica.

González, V. P. (2015). La teoría del color en fomento del aprendizaje de los niños con discapacidad auditiva del tercer año básico de la escuela municipal de audición y lenguaje. (Tesis Universidad de Guayaquil). Facultad de Comunicación Social. Carrera de Ingeniería en Diseño Gráfico.

Gutiérrez, R. (1998). La Estética del Espacio Escolar. Barcelona, España: Oikos-Tau.

López, R. J., y Gauchi, J. M. (2009). Estrategias de comunicación corporativa en las franquicias de restauración. Análisis cromático de la identidad visual. Revista Latina de Comunicación Social, (64), 1-12. doi: org.ezproxy.sibdi.ucr.ac.cr/10.4185/RLCS-64-2009-824-300-314

Naranjo, P. M. (2010). Factores que favorecen el desarrollo de una actitud positiva hacia las actividades académicas. Revista de Educación, 34(1), 31-53.

Padilla, F. (1998). Respuesta del Edifico Escolar al Uso y Solicitaciones (Análisis del deterioro), Énfasis en el Valle Central, Propuesta de Mejoras. (Tesis de Licenciatura). Universidad de Costa Rica, Costa Rica.

Palacios, M. L., y Zaraza, L. J. (2013). Relación que Existe entre el Espacio Escolar y las Experiencias Significativas en los Niños y Niñas del Grado Transición del Jardín Infantil la Gacela. (Tesis de Licenciatura). Universidad de San Buenaventura Seccional Medellín, Medellín. Recuperado de https://bit.ly/2PgT2V3

Santos, G. M. (1993.). Espacios escolares. Recuperado de https://bit.ly/2E24CRT

Sanz, A. (2016). Factores estéticos determinantes de la calidad y el confort en el aula infantil. Revista Electrónica Interuniversitaria de Formación del Profesorado,19(3), 53-65. doi: http://dx.doi.org/10.6018/ reifop.19.3.267241

Sevilla, K., Sanabria, J., y Shedden, M. (2010). Compendio de normas y recomendaciones para la construcción de edificios para la educación (DIEE-MEP). Costa Rica: Ministerio de Educación Pública, Dirección de Infraestructura y Equipamiento Educativo.

Viñao, A. (1993). El Espacio Escolar Introducción. Revista Historia de la educación, 12. Recuperado de https:// bit.ly/2zII31s

Yebra, M., Bleda, S., y Vera, J. (2002). Necesidad de pautas y normativa acústica especificas en las construcciones escolares: La Universidad de Alicante, un ejemplo. Departamento de Física. Recuperado de: https://bit.ly/2SBz2yD

\section{BY-NC-ND}

